\title{
ON A CLASS OF QUADRATIC ALGEBRAS
}

\author{
R. D. SCHAFER
}

In a recent paper [3] concerning cubic forms which permit a new type of composition, a class of nonassociative algebras $A$ with 1 over $F$ of characteristic $\neq 2$ is encountered: central simple flexible quadratic algebras satisfying the identity

$$
[x,[x, y]]=0 \quad \text { for all } x, y \text { in } A .
$$

We shall denote this class by $\mathfrak{C}$. The commutative algebras in $\mathfrak{C}$ are the well-known central simple Jordan algebras of degree two. In [3] an example is given (over $F$ containing $\sqrt{ }-1$ of a 7 -dimensional algebra in $\mathfrak{C}$ which is not commutative. We shall denote by $\mathfrak{C}^{\prime}$ the class of all algebras in $\mathfrak{e}$ which are not commutative.

In this note we show that, in the presence of the other conditions on the algebras in $\mathfrak{C}$, identity (1) may be replaced by

$$
[x, y]^{2}=0 \quad \text { for all } x, y \text { in } A \text {. }
$$

For arbitrary $F$ of characteristic $\neq 2$ and for any dimension $\geqq 7$ (possibly infinite), we show that $\mathfrak{e}^{\prime}$ contains an algebra $A$ of dimension $n$ over $F$. On the other hand, if $F$ is also of characteristic $\neq 3$, then $\mathfrak{e}^{\prime}$ contains no algebra of dimension $\leqq 6$.

An algebra $A$ (of possibly infinite dimension) with 1 over a field $F$ is called a quadratic algebra in case $A \neq F 1$ and, for every $x$ in $A$, there are $t(x), n(x)$ in $F$ such that

$$
x^{2}-t(x) x+n(x) 1=0 .
$$

It is well-known that, by defining $t(\alpha 1)=2 \alpha, n(\alpha 1)=\alpha^{2}$, the trace $t(x)$ is linear and the norm $n(x)$ is a quadratic form. If $F$ has characteristic $\neq 2$, there is a (possibly infinite) basis $U=\left\{u_{i}\right\}$ of $A$ over $F$ such that $1=u_{0} \in U$,

$$
u_{i}^{2}=\beta_{i} 1, \quad \quad \beta_{i} \in F, \beta_{0}=1,
$$

and, for $i \neq 0, j \neq 0, i \neq j$,

$$
u_{i} u_{j}=\sum \pi_{i j k} u_{k}=-u_{j} u_{i} \quad(i \neq j ; i \neq 0, j \neq 0)
$$

where only a finite number of the $\pi_{i j k}$ are $\neq 0$. From (5) we have

$$
\pi_{i j k}=-\pi_{j i k} \text { for all } k \quad(i \neq j ; i \neq 0, j \neq 0) .
$$

Presented to the Society, April 8, 1961; received by the editors February 27, 1961. 
Any $x \in A$ may be written uniquely in the form

$$
x=\sum \alpha_{i} u_{i}
$$

with only a finite number of the $\alpha_{i} \neq 0$. Then

$$
t(x)=2 \alpha_{0}, \quad n(x)=\alpha_{0}^{2}-\sum_{i \neq 0} \alpha_{i}^{2} \beta_{i} .
$$

It is well-known that $n(x)$ is nondegenerate if and only if in (4) we have $\beta_{i} \neq 0$ for every $i$. One sees easily $[2 ; 3]$ that $A$ is central simple if and only if $n(x)$ is nondegenerate and the dimension of $A$ is $\geqq 3$. Clearly $A$ is commutative if and only if all $\pi_{i j k}=0$ in (5).

An algebra $A$ over $F$ is called flexible in case

$$
(x y) x=x(y x) \quad \text { for all } x, y \text { in } A .
$$

It is shown in $[1$, p. 588] that a quadratic algebra is flexible if and only if

$$
\pi_{i j 0}=\pi_{i j i}=\pi_{i j j}=0 \quad(i \neq j ; i \neq 0, j \neq 0)
$$

and

$$
\beta_{i} \pi_{j k i}=\beta_{k} \pi_{i j k} \quad(i, j, k \text { distinct } ; \neq \neq 0, j \neq 0, k \neq 0)
$$

are satisfied in (5). Hence the algebras in $\mathcal{C}$ are the algebras $A$ of dimension $\geqq 3$ over $F$ of characteristic $\neq 2$ satisfying (4) with all $\beta_{i} \neq 0,(5),(6),(10),(11)$, and (1) where $[x, y]$ is the commutator $[x, y]=x y-y x$. The algebras in $\mathfrak{C}^{\prime}$ satisfy the additional requirement that at least one $\pi_{i j k}$ in (5) is $\neq 0$.

Let $(x, y)$ be the nondegenerate symmetric bilinear form such that $n(x)=(x, x)$. In [3, equation (78)] it is shown that, for any $A$ in $\mathfrak{e}$, we have

$$
n(x y)=(x y, y x)=n(y x) \quad \text { for all } x, y \text { in } A .
$$

Hence $n([x, y])=(x y-y x, x y-y x)=n(x y)-2(x y, y x)+n(y x)=0$ for all $x, y$ in $A$. Since also $t([x, y])=0[3$, p. 172], we see that (3) implies (2). But then we may replace (1) by (2) in the definition of $\mathfrak{C}$. For, conversely, we may linearize (2) in $y$ to obtain

$$
[x, y][x, z]+[x, z][x, y]=0 .
$$

In $[3$, p. 172] it is shown that flexibility implies

$$
t(x y)=t(y x), \quad t((x y) z)=t(x(y z)),
$$

and 


$$
(x, y)=\frac{1}{2} t(x \bar{y}) \quad \text { where } \quad \bar{y}=t(y) 1-y .
$$

Hence (12) implies

$$
\begin{aligned}
0 & =t([x, y][x, z]+[x, z][x, y]) \\
& =2 t([x, y][x, z]) \\
& =2 t((x y)(x z)-(z x)(x y)-(y x)(x z)+(z x)(y x)) \\
& =2 t((x y x) z-z(x(x y))-((y x) x) z+z(x y x)) \\
& =(2 x y x-x(x y)-(y x) x, \bar{z})
\end{aligned}
$$

for every $z$ in $A$. Since $(x, y)$ is nondegenerate, we have $2 x y x-x(x y)$ $-(y x) x=0$, implying (1).

Although (1) has the advantage of being an identity of lower degree than identity (2), the latter may be very easily stated: the square of every commutator is 0 . We shall use this in the proof of the Theorem below.

Lemma. Let $A_{1}$ over $F$ be an algebra in $\mathfrak{e}^{\prime}$, and let the dimension of $A_{1}$ be $m$. Then, for any $n \geqq m$ (possibly infinite), there exists an $n$ dimensional algebra $A$ over $F$ in $\mathfrak{C}^{\prime}$ such that $A_{1}$ is a subalgebra of $A$.

Proof. A basis $U_{1}$ for $A_{1}$ (of the special form we have chosen) may be extended to a basis $U=U_{1} \cup U_{2}$ for an algebra $A$ over $F$ such that $A$ has dimension $n$ over $F$. It remains to define multiplication appropriately for pairs of elements in $U$ where at least one of the elements of the pair is in $U_{2}$. Define

$$
u_{i}^{2}=-1 \quad \text { for } u_{i} \text { in } U_{2},
$$

and

$$
u_{i} u_{j}=0 \quad \text { for } i \neq j \text {, if either } u_{i} \text { or } u_{j} \text { is in } U_{2} \text {. }
$$

All of the conditions for $A$ to be in $\mathfrak{e}^{\prime}$ are obviously satisfied, except possibly for (11) and (2) which we verify as follows. If $u_{i}, u_{j}$, and $u_{k}$ are all in $U_{1}$, then (11) is satisfied since $A_{1}$ is in $\mathcal{C}$. If both $u_{i}$ and $u_{j}$ are in $U_{1}$, then $u_{i} u_{j}$ in $A_{1}$ implies $\pi_{i j k}=0$ for all $k$ for which $u_{k} \in U_{2}$; hence (14) implies (11) in this case. Finally, if either $u_{i}$ or $u_{j}$ is in $U_{2}$, then $\pi_{i j k}=\pi_{j k i}=0$ by (14). To verify (2), we write $x$ in $A$ in the form $x=x_{1}+x_{2}$ where $x_{1} \in A_{1}$ and where $x_{2}$ is a linear combination of elements of $U_{2}$. With $y=y_{1}+y_{2}$ written similarly, we obtain $[x, y]$ $=\left[x_{1}, y_{1}\right]$ by (14). Then $[x, y]^{2}=\left[x_{1}, y_{1}\right]^{2}=0$ since $A_{1}$ is in $\mathrm{e}$.

THEOREM. Let $F$ be an arbitrary field of characteristic $\neq 2$. For any dimension $n \geqq 7$ (possibly infinite), there exists an $n$-dimensional algebra 
$A$ over $F$ in the class $\mathfrak{C}^{\prime}$. If $F$ is also of characteristic $\neq 3$, there are no algebras $A$ of dimension $\leqq 6$ over $F$ in $\mathfrak{C}^{\prime}$.

Proof. We use the lemma to see that (i) the first statement in the conclusion of the Theorem may be established by constructing a 7-dimensional algebra in $\mathfrak{C}^{\prime}$, while (ii) the final statement may be proved by showing that any 6-dimensional algebra in $\mathfrak{C}$ is commutative (assuming characteristic $\neq 3$ ).

To construct an example in (i), let $1, u_{1}, u_{2}, \cdots, u_{6}$ be a basis for $A$ over $F$. Define

$$
u_{i}^{2}=-1, \quad u_{i+3}^{2}=1, \quad u_{i} u_{i+3}=u_{i+3} u_{i}=0 \quad(i=1,2,3) .
$$

For cyclic permutations $i, j, k$ of $1,2,3$, define

$$
\begin{aligned}
u_{i} u_{j} & =u_{i} u_{j+3}=u_{i+3} u_{j}=u_{i+3} u_{j+3}=u_{k}-u_{k+3}=-u_{j} u_{i} \\
& =-u_{j+3} u_{i}=-u_{j} u_{i+3}=-u_{j+3} u_{i+3} .
\end{aligned}
$$

Then (4) with all $\beta_{i} \neq 0$, (5), (6), (10) and (11) are satisfied. In order to verify (1) we note that, relative to the basis $1, u_{1}, u_{2}, \cdots, u_{6}$, the matrix $T_{x}$ of the linear transformation $y \rightarrow[x, y] / 2$ has the form

$$
T_{x}=\left(\begin{array}{ccc}
0 & 0 & 0 \\
0 & K & -K \\
0 & K & -K
\end{array}\right)
$$

where

$$
K=\left(\begin{array}{ccc}
0 & -\alpha_{3}-\alpha_{6} & \alpha_{2}+\alpha_{5} \\
\alpha_{3}+\alpha_{6} & 0 & -\alpha_{1}-\alpha_{4} \\
-\alpha_{2}-\alpha_{5} & \alpha_{1}+\alpha_{4} & 0
\end{array}\right) \quad \text { for } x \text { in (7). }
$$

Hence $T_{x}^{2}=0$, implying (1).

To prove (ii), we may as well assume that $F$ is algebraically closed. For, if $\Sigma$ is the algebraic closure of $F$, and if $A$ over $F$ is in the class $\mathfrak{C}^{\prime}$, then $A_{\Sigma}$ is in the corresponding class of algebras over $\Sigma$ (the equations (4), (5), (6), (10), and (11) remain unchanged, and one may linearize (1) to obtain an equivalent multilinear identity). Hence we may assume that $\beta_{i}=-1(i=1, \cdots, 5)$ in (4). The 60 elements $\pi_{i j k}$ which are not given as 0 by (10) are partitioned by the relations (6) and (11) into 10 classes, within each of which the elements differ only by a factor of \pm 1 . Writing subscripts modulo 5 (using 5 , not 0 ), we make the following abbreviations for representatives of these 10 classes: 


$$
\sigma_{i}=\pi_{i+1, i+2, i+3}, \quad \epsilon_{i}=\pi_{i+1, i+3, i+4} \quad(i=1, \cdots, 5) .
$$

Then, for subscripts modulo 5 , we have

$$
u_{i} u_{i+1}=\sigma_{i+4} u_{i+2}+\epsilon_{i+2} u_{i+3}+\sigma_{i+3} u_{i+4} \quad(i=1, \cdots, 5)
$$

and

$$
u_{i} u_{i+3}=-\epsilon_{i+2} u_{i+1}-\epsilon_{i+4} u_{i+2}+\sigma_{i+2} u_{i+4} \quad(i=1, \cdots, 5) .
$$

Now (2), (5) and (4) imply $\left[u_{i}, u_{j}\right]^{2}=\left(2 u_{i} u_{j}\right)^{2}=-4 \Sigma \pi_{i j k}^{2}=0$ for $i \neq j$. Hence (16) implies

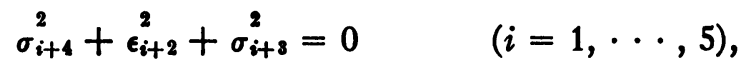

and (17) implies

$$
\epsilon_{i+2}^{2}+\underset{\epsilon_{i+4}^{2}}{2}+\sigma_{i+2}^{2}=0 \quad(i=1, \cdots, 5) .
$$

Eliminating the $\epsilon$ 's in (18) and (19), and adding the resulting equations, we arrive easily at $\sigma_{i}=\epsilon_{i}=0(i=1, \cdots, 5)$ since the characteristic of $F$ is $\neq 2,3$. Hence $A$ is commutative.

REMARK. Characteristic $\neq 3$ is not required in a direct proof that there are no algebras in $\mathfrak{e}^{\prime}$ of dimension $\leqq 5$.

\section{REFERENCES}

1. A. A. Albert, Power-associative rings, Trans. Amer. Math. Soc. 64 (1948), 552593.

2. Nathan Jacobson, Structure of alternative and Jordan bimodules, Osaka Math. J. 6 (1954), 1-71.

3. R. D. Schafer, Cubic forms permitting a new type of composition, J. Math. Mech. 10 (1961), 159-174.

Massachusetts Institute of Technology 